\title{
Mass cytometry detects H3.3K27M-specific vaccine responses in diffuse midline glioma
}

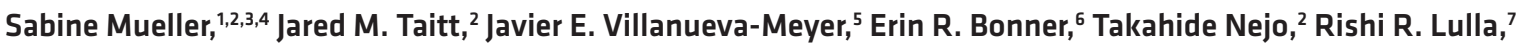 \\ Stewart Goldman, ${ }^{8}$ Anu Banerjee, ${ }^{2,3}$ Susan N. Chi, ${ }^{9}$ Nicholas S. Whipple, ${ }^{10}$ John R. Crawford, ${ }^{11}$ Karen Gauvain, ${ }^{12}$ Kellie J. Nazemi, ${ }^{13}$ \\ Payal B. Watchmaker, ${ }^{2}$ Neil D. Almeida, ${ }^{14}$ Kaori Okada, ${ }^{2}$ Andres M. Salazar, ${ }^{15}$ Ryan D. Gilbert, ${ }^{2}$ Javad Nazarian, ${ }^{4,6}$ \\ Annette M. Molinaro, ${ }^{2}$ Lisa H. Butterfield, ${ }^{16,17}$ Michael D. Prados, ${ }^{2,3}$ and Hideho Okada ${ }^{2,16,18}$

\begin{abstract}
'Department of Neurology, ${ }^{2}$ Department of Neurosurgery and ${ }^{3}$ Department of Pediatrics, UCSF, San Francisco, California, USA. "Children's University Hospital Zurich, Switzerland. ${ }^{4}$ Department of Radiology and Biomedical Imaging, UCSF, San Francisco, California, USA. ${ }^{6}$ Children's National Medical Center, Washington, DC, USA. Division of Pediatric Hematology/Oncology, Hasbro Children's Hospital, Department of Pediatrics, The Warren Alpert Medical School of Brown University, Providence, Rhode Island, USA. ${ }^{8}$ Ann \& Robert H. Lurie Children's Hospital of Chicago, Chicago, Illinois, USA. ㅁDana-Farber Cancer Institute, Boston, Massachusetts, USA. ${ }^{10}$ Division of Hematology/Oncology, Department of Pediatrics, University of Utah, Salt Lake City, Utah, USA. "Department of Neurosciences and Pediatrics, UCSD and Rady Children's Hospital, San Diego, California, USA. ${ }^{2}$ St. Louis Children's Hospital, Washington University in St. Louis, St. Louis, Missouri, USA. ${ }^{13}$ Doernbecher Children's Hospital, Oregon Health \& Science University, Portland, Oregon, USA. ${ }^{14}$ The George Washington University School of Medicine and Health Sciences, The George Washington University, Washington, District of Columbia, USA. ${ }^{15}$ Oncovir Inc., Washington, DC, USA. ${ }^{16}$ Parker Institute for Cancer Immunotherapy, San Francisco, California, USA. ${ }^{17}$ Department of Microbiology and Immunology, UCSF, San Francisco, California, USA. ${ }^{18}$ Helen Diller Family
\end{abstract} \\ Comprehensive Cancer Center, UCSF, San Francisco, California, USA.
}

BACKGROUND. Patients with diffuse midline gliomas (DMGs), including diffuse intrinsic pontine glioma (DIPG), have dismal outcomes. We previously described the H3.3K27M mutation as a shared neoantigen in HLA-A*02.01+, H3.3K27M+ DMGs. Within the Pacific Pediatric Neuro-Oncology Consortium, we assessed the safety and efficacy of an H3.3K27M-targeted peptide vaccine.

METHODS. Newly diagnosed patients, aged 3-21 years, with $\mathrm{HLA}-\mathrm{A}^{*} 02.01^{+}$and $\mathrm{H3} .3 \mathrm{~K} 27 \mathrm{M}^{+}$status were enrolled in stratum $\mathrm{A}$ (DIPG) or stratum B (nonpontine DMC). Vaccine was administered in combination with polyinosinic-polycytidylic acid-poly-IIysine carboxymethylcellulose (poly-ICLC) every 3 weeks for 8 cycles, followed by once every 6 weeks. Immunomonitoring and imaging were performed every 3 months. Imaging was centrally reviewed. Immunological responses were assessed in PBMCs using mass cytometry.

RESULTS. A total of 19 patients were enrolled in stratum A (median age,11 years) and 10 in stratum B (median age, 13 years). There were no grade- 4 treatment-related adverse events (TRAEs). Injection site reaction was the most commonly reported TRAE. Overall survival (OS) at 12 months was $40 \%$ (95\% Cl, 22\%-73\%) for patients in stratum A and $39 \%(95 \% \mathrm{Cl}, 16 \%-93 \%)$ for patients in stratum B. The median OS was 16.1 months for patients who had an expansion of H3.3K27M-reactive CD8 ${ }^{+} \mathrm{T}$ cells compared with 9.8 months for their counterparts $(P=0.05)$. Patients with DIPC with below-median baseline levels of myeloid-derived suppressor cells had prolonged OS compared with their counterparts $(P<0.01)$. Immediate pretreatment dexamethasone administration was inversely associated with H3.3K27M-reactive CD8+ $\mathrm{T}$ cell responses.

CONCLUSION. Administration of the H3.3K27M-specific vaccine was well tolerated. Patients with H3.3K27M-specific CD8 immunological responses demonstrated prolonged OS compared with nonresponders.

TRIAL REGISTRATION. ClinicalTrials.gov NCT02960230.

FUNDING. The V Foundation, the Pacific Pediatric Neuro-Oncology Consortium Foundation, the Pediatric Brain Tumor Foundation, the Mithil Prasad Foundation, the MCJ Amelior Foundation, the Anne and Jason Farber Foundation, Will Power Research Fund Inc., the Isabella Kerr Molina Foundation, the Parker Institute for Cancer Immunotherapy, and the National Institute of Neurological Disorders and Stroke (NINDS), NIH (R35NS105068).

Authorship note: SM and JMT contributed equally to this work.

Conflict of interest: $\mathrm{HO}$ is an inventor of a utility patent application titled "H3.3 CTL peptides and uses thereof" (attorney docket no: 81906-938904-220400 US,

client reference no. SF15-163), which has been exclusively licensed to Tmunity Therapeutics Inc.

Copyright: (c) 2020, American Society for Clinical Investigation.

Submitted: May 29, 2020; Accepted: August 11, 2020; Published: October 26, 2020.

Reference information: / Clin Invest. 2020;130(12):6325-6337.

https://doi.org/10.1172/JCl140378.

\section{Introduction}

Diffuse midline gliomas (DMGs) are uniformly fatal pediatric brain cancers, and despite maximal therapy, survival outcomes remain dismal, with less than $10 \%$ of patients surviving beyond 2 years (1). A hallmark of DMG is a set of recurrent mutations in genes encoding histone $\mathrm{H} 3$ variants including histones $\mathrm{H} 3.3$ (H3F3A) and H3.1 (HIST1H3B and HIST1H3C) (2, 3). Recently, immunotherapy has garnered significant attention as a treatment 


\section{Table 1. Patients' baseline characteristics}

\begin{tabular}{|c|c|c|c|}
\hline Characteristics & Whole cohort $(n=29)$ & DIPG (stratum A; $n=19$ ) & Other DMG (stratum B; $n=10$ ) \\
\hline Median age, yr (range) & $11(5-18)$ & $11(5-17)$ & $12(7-18)$ \\
\hline Female, no. (\%) & $16(55 \%)$ & $10(53 \%)$ & $6(60 \%)$ \\
\hline Male, no. $(\%)$ & $13(45 \%)$ & $9(47 \%)$ & $4(40 \%)$ \\
\hline Pons & 21 & 19 & 2 (with cerebellar/spinal cord) \\
\hline Thalamic & 6 & 0 & 7 \\
\hline White & 18 & 11 & 7 \\
\hline African American & 2 & 1 & 1 \\
\hline Other (not available) & 8 & 7 & 1 \\
\hline Declined to identify & 1 & 0 & 1 \\
\hline \multicolumn{4}{|l|}{ Ethnicity } \\
\hline Median no. of days from end of RT to first vaccine (range) & $40(22-71)$ & $39(27-71)$ & $39(22-53)$ \\
\hline Median no. of vaccines (range) & $6(1-11)$ & $7(2-11)$ & $5(1-11)$ \\
\hline Positive H3.3K27M detection in plasma (\% of available samples) & $41 / 60(68.3 \%)$ & $29 / 42(69.0 \%)$ & $12 / 18(66.7 \%)$ \\
\hline PBMC immune profiling, median no. of samples per patient (range) & $2(0-5)^{B}$ & $2(1-5)$ & $2(0-3)$ \\
\hline Steroid use, no. (\%) & $13(45)$ & $9(47)$ & $4(40)$ \\
\hline Temozolomide use during RT, no. (\%) & $4(13.8 \%)$ & $3(15.8 \%)$ & $1(10 \%)$ \\
\hline \multicolumn{4}{|l|}{ Off-study treatment criteria } \\
\hline Progression & 27 & 18 & 9 \\
\hline Lost to follow-up; withdrawal of consent & 1 & 0 & 1 \\
\hline Toxicity & 1 & 1 & 0 \\
\hline
\end{tabular}

${ }^{A}$ Excluding 1 patient who had biopsy after start of radiation therapy. ${ }^{B}$ One patient sample (PNOC007-16) lost due to technical difficulties during data acquisition.

for cancers harboring tumor-specific neoantigens. We have identified a 10 mer peptide spanning position $26-35\left(\mathrm{H} 3.3 \mathrm{~K}_{2} 7 \mathrm{M}_{26-35}\right)$ of the $\mathrm{H} 3.3 \mathrm{~K} 27 \mathrm{M}$ protein as an $\mathrm{HLA}^{*} \mathrm{~A}^{*}$ 2:01-restricted cytotoxic T lymphocyte (CTL) epitope (4). Although most missense mutationderived neoantigens are unique to individual patients (5), a majority of patients with DMG and more than $70 \%$ of patients with diffuse intrinsic pontine glioma (DIPG) have the H3.3K27M mutation (6). The H3.3K27M-derived neoantigen epitope represents a valuable target, as it is not only tumor specific, but also K27Mmutant protein is present throughout all tumor nuclei in each of the 47 cases evaluated by immunohistochemistry (7), suggesting that this may be a truncal mutation and less likely to lead to antigen lossmediated escape from the tumor. Within this multicenter study conducted through the Pacific Pediatric Neuro-Oncology Consortium (PNOC), we evaluated the safety, immunoreactivity, and efficacy of the synthetic $\mathrm{H} 3.3 \mathrm{~K} 27 \mathrm{M}_{26-35}$ peptide in combination with the helper tetanus toxoid (TT) peptide and polyinosinicpolycytidylic acid-poly-I-lysine carboxymethylcellulose (poly-ICLC) adjuvant in patients with HLA-A ${ }^{*} 2: 01^{+}, \mathrm{H} 3.3 \mathrm{~K} 27 \mathrm{M}^{+}$DMGs.

\section{Results}

Patient characteristics. From November 2016 until March 2019, a total of 19 eligible patients (median age, 11 years; $\mathrm{IQR}=4.5$ years; $53 \%$ female, $47 \%$ male) were enrolled in stratum A and 10 eligible patients (median age, 13 years; $I Q R=4.25$ years; $60 \%$ female,
$40 \%$ male) in stratum B (Table 1). DMGs in stratum B included 7 thalamic tumors, 2 pontine-centered tumors that did not meet the imaging criteria for DIPGs, with extension into the cerebellum in both cases and the cervical spine in 1 , and 1 spinal cord tumor located within the thoracic spine. The median time between tumor tissue collection and the start of radiation therapy (RT) was 17 days (range, 4-25 days) for patients in stratum A and 22 days (range, 2-49 days) for those in stratum B, except for 1 patient, who had a biopsy performed after RT completion. The median time from completion of RT to the first vaccine was 39 days (range, 27-71 days) for patients in stratum $A$ and 39 days (range, 22-53 days) for patients in stratum B.

Treatment and safety. The median number of vaccinations administered was $7(\mathrm{IQR}=4)$ and $5(\mathrm{IQR}=4.5)$ for strata $\mathrm{A}$ and $\mathrm{B}$, respectively. Treatment was generally well tolerated with no grade 4 treatment-related adverse events (TRAEs). The most common TRAE was injection site reaction (Table 2). One patient from stratum A experienced a regimen-limiting toxicity (RLT), presenting with signs and symptoms of meningitis. This patient was treated according to institutional guidelines with antibiotics and was hospitalized until recovery. Blood and cerebrospinal fluid remained negative for meningitis, although the patient was started on antibiotics before sample collection. After recovery from this event, the patient was taken off the study, as TRAEs could not be excluded. There were no other instances of meningitis. 
Table 2. H3.3K27M/TT-related AEs ${ }^{A}$

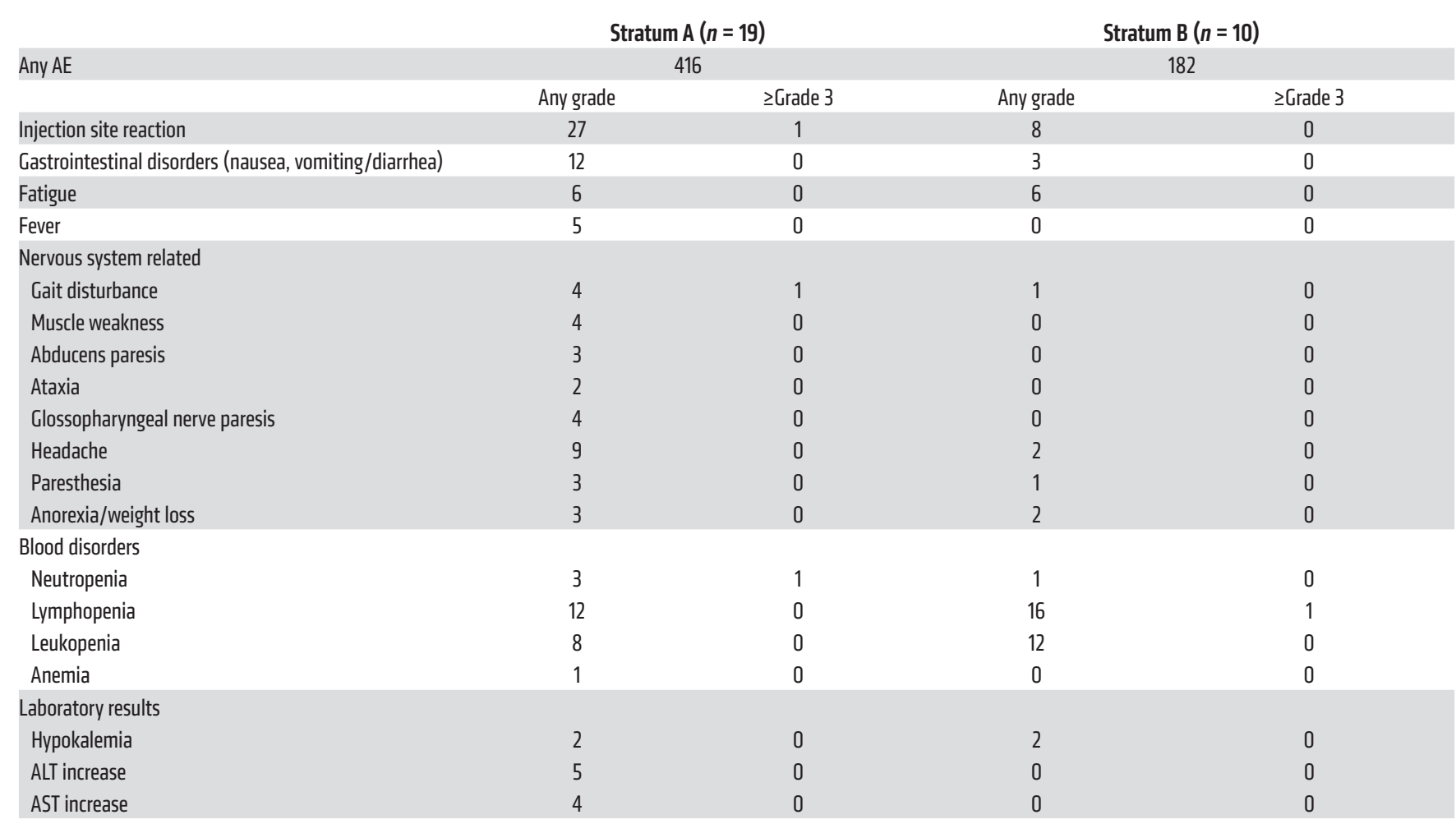

${ }^{A}$ Occurring in more than 1 subject.

Clinical efficacy. The primary efficacy endpoint was the overall survival at 12 months (OS12) of patients in stratum A. With a median follow-up time of 17.6 months, the OS12 rate was $44 \%$ (95\% CI, $27 \%-75 \%)$ for patients in stratum A and 39\% (95\% CI, 16\%-93\%) for those in stratum B (Figure 1A). The median progression-free survival (PFS) was 4.9 months (95\% CI, 3-8.3 months) for stratum A and 3.5 months (95\% CI, 3-NA) for stratum B (Figure 1B). One patient in stratum A had a positive response (partial response; PNOC007-25).

Detection, validation, clustering, and phenotyping of H3.3K27Mreactive $C D 8^{+} T$ cells. CyTOF-based immune analyses were conducted on 28 of the total 29 patients enrolled in the study, in which H3.3K27M-reactive $\mathrm{CD}^{+} \mathrm{T}$ cells were detected using a conventional $\mathrm{CD}^{+}$gating strategy (Supplemental Figure 1; supplemental material available online with this article; https://oi.org/10.1172/ JCI140378DS1). H3.3K27M dextramer staining revealed negligible nonspecific staining on healthy donor-derived PBMCs (Figure 1C). CyTOF-based H3.3K27M-reactive CD8 ${ }^{+} \mathrm{T}$ cell detection was determined to be as sensitive as flow cytometry (Supplemental Figure 2). We observed that $\mathrm{H} 3.3 \mathrm{~K} 27 \mathrm{M}$-reactive $\mathrm{CD} 8^{+} \mathrm{T}$ cells were clustered on a t-distributed stochastic neighbor embedding (t-SNE) plot and grouped into 5 subpopulations (Supplemental Figure 3): early activated (Tea), stem cell memory (Tscm), effector (Teff), effector memory (Tem), and exhausted (Tex) phenotypes based on their aggregate phenotypic profiles (Figure 1D and Supplemental Figure 3).

Expansion of $\mathrm{H3} 3.3 \mathrm{~K} 27 \mathrm{M}$-reactive $\mathrm{CD} 8^{+} \mathrm{T}$ cells is associated with a better prognosis. Of the patients from whom PBMCs were col- lected at multiple time points $(n=18)$, we analyzed longitudinal H3.3K27M-reactive $\mathrm{CD} 8^{+} \mathrm{T}$ cell frequencies (Supplemental Figure 4), which revealed that $7(39 \%)$ patients met the criteria for an immunological response (Figure 1E). Among these patients, the median OS of overall immunological responders was 16.3 months $(n=7,95 \%$ CI, $12.6-\mathrm{NA})$ compared with 9.9 months $(n=11,95 \%$ CI, 9.0-NA) for nonresponders ( $P=0.05$, log-rank test) (Figure $1 F$ ), with an estimated multivariate HR of 0.04 (95\% CI, 0.004$0.045, P<0.01$ ) (Supplemental Table 1). Further, immunological responders exhibited prolonged PFS ( $P=0.05$, log-rank test) compared with nonresponders (Figure $1 G)$. Patients with Tem $(n=6$, $P=0.02, \log$-rank test) expansions also exhibited trends toward prolonged OS (Figure $1 \mathrm{H}$ and Supplemental Figure 5) and PFS ( $P$ $=0.06$, log-rank test) compared with their counterparts (Figure 1I and Supplemental Figure 6). These trends persisted when controlling for stratum, as patients with DIPG showing overall immunological responses had a median OS of 16.1 months $(n=6,95 \%$ CI, 12.4-NA) compared with 10 months ( $n=6,95 \% \mathrm{CI}, 9.5-\mathrm{NA})$ for nonresponders ( $P=0.11, \log$-rank) (Supplemental Figure 7), with an estimated multivariate $\mathrm{HR}$ of 0.03 (95\% CI, 0.001 to 1.960 , $P=0.10$ ) (Supplemental Table 1). Prolonged OS among patients with DIPG with Tem expansions ( $n=6, P=0.03$, log-rank) further strengthened this association, while also being substantiated by prolonged PFS $(P<0.01$, log-rank) relative to nonresponders (Supplemental Figure 8). These corroborating survival trends did not persist when using the same longitudinal immunological response threshold for bulk $\mathrm{CD} 8^{+} \mathrm{T}$ cell phenotypic subtypes (Supplemental 
A

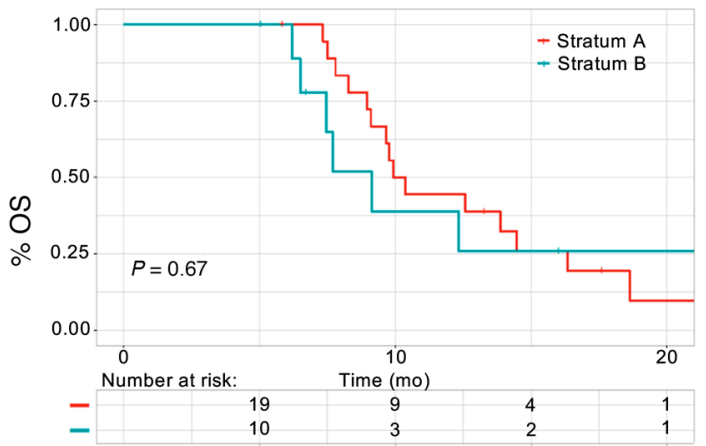

C

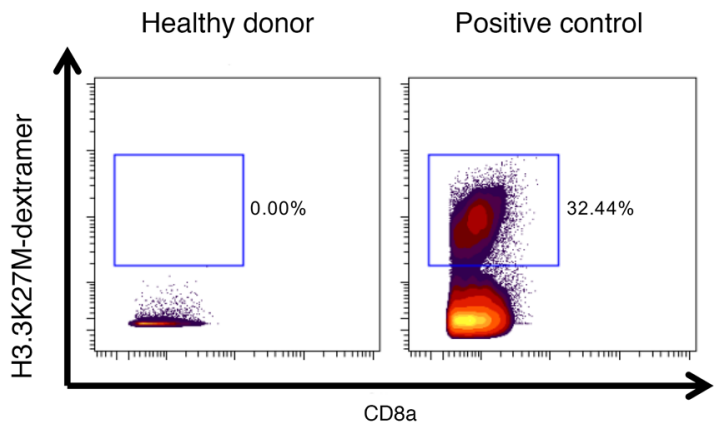

B

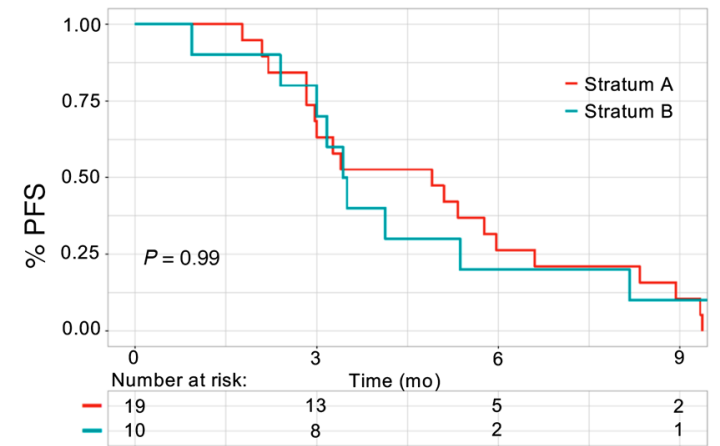

D

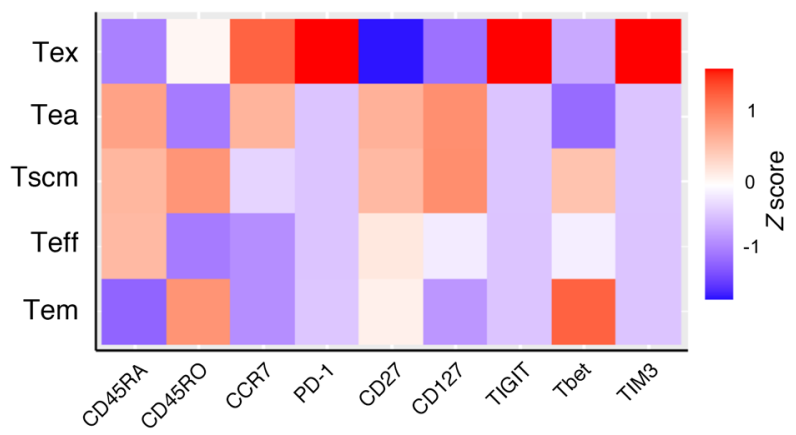

$\mathbf{E}$

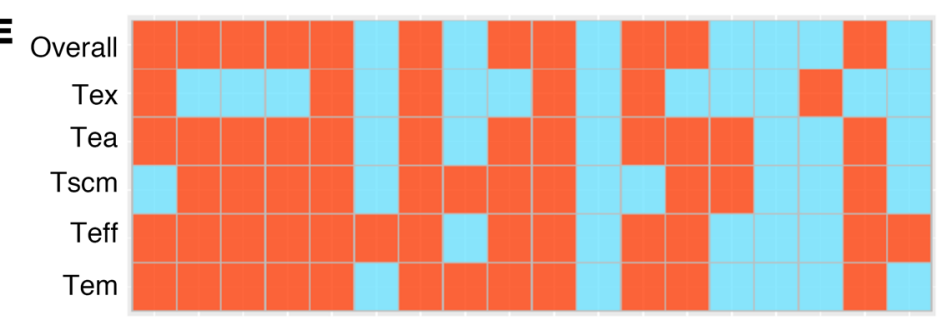

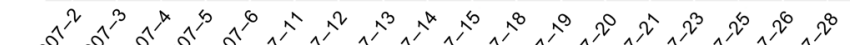

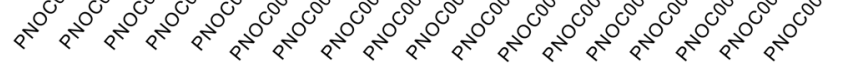

$\mathbf{F}$

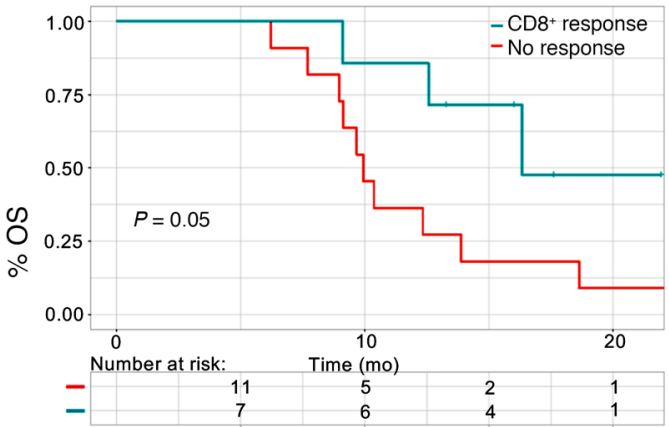

H

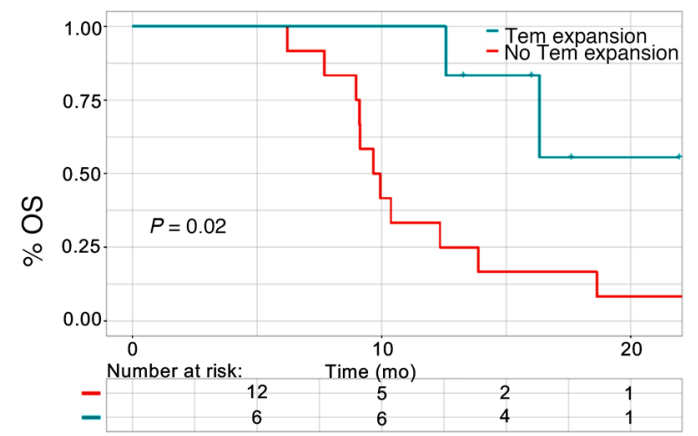

G

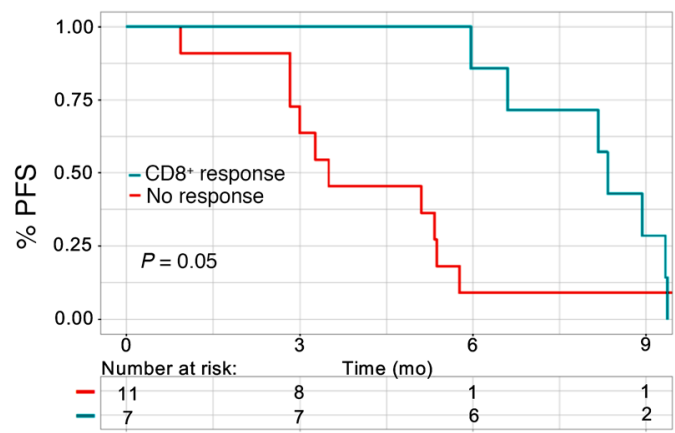

I

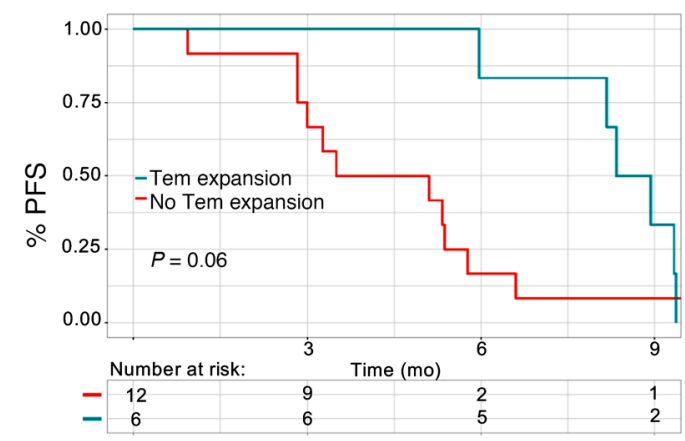


Figure 1. Mass cytometry-based phenotyping of H3.3K27M-reactive $\mathrm{CD8}^{+} \mathrm{T}$ cell subpopulations reveals associations between immunological responses and prolonged OS or PFS. (A and B) Kaplan-Meier survival curves contrasting the (A) OS and (B) PFS of patients enrolled in stratum A (red) and stratum B (blue) using log-rank tests. (C) Left: PBMCs derived from a healthy donor showed an absence of H3.3K27M dextramer staining. Right: The H3.3K27M dextramer exhibited sensitive detection of H3.3K27Mspecific T cell receptor-transduced (TCR-transduced) CD8 ${ }^{+} \mathrm{T}$ cells. (D) Heatmap visualizing the relative expression ( $Z$ score) of subpopulation markers in patient-derived $\mathrm{H} 3.3 \mathrm{~K} 27 \mathrm{M}$-reactive $\mathrm{CD}^{+} \mathrm{T}$ cells. (E) Heatmap visualizing the patient-specific presence (blue) or absence (red) of an expansion of H3.3K27M-reactive CD8 ${ }^{+}$T cells in a subpopulation-specific manner. (F and $\mathbf{G})$ Kaplan-Meier survival curves contrasting the (F) OS and (C) PFS of patients who had an immunological response (blue) compared with patients who did not (red) using log-rank tests. (H and I) Kaplan-Meier survival curves contrasting the (H) OS and (I) PFS of patients who had an expansion of effector memory H3.3K27M-reactive CD8 ${ }^{+} \mathrm{T}$ cells (blue) compared with patients who did not (red) using log-rank tests.

Figures 9-12), suggesting that the survival benefit associated with the expansion of $\mathrm{H} 3.3 \mathrm{~K} 27 \mathrm{M}$-reactive $\mathrm{CD}^{+} \mathrm{T}$ cells is independent of overall T cell status. No association between H3.3K27M-reactive Tex expansion and patient outcomes was established (Supplemental Figures 5-8). The number of vaccines administered correlated with the longitudinal change in the percentage of H3.3K27Mreactive $\mathrm{CD}^{+} \mathrm{T}$ cells at the final time point analyzed relative to the percentage at baseline (Supplemental Figure 13). However, the median number of vaccines preceding immunological responses was $6(\mathrm{IQR}=2)$ among responders, whereas the median number of total vaccines was also $6(\mathrm{IQR}=4)$ among the nonresponders (Figure 2), suggesting that the number of vaccines is not the sole factor for separating responders from nonresponders.

Circulatory MDSC abundance at baseline functions as a prognostic indicator for patients with DIPG. We identified myeloid cells using a conventional myeloid gating strategy (Figure 3A). Of these clusters, early myeloid-derived suppressor cells (E-MDSCs) $\left(\mathrm{CD} 33^{+} \mathrm{CD} 11 \mathrm{~b}^{+} \mathrm{HLA}-\mathrm{DR}^{\mathrm{lo}} \mathrm{Lin}^{\mathrm{lo}}\right)$ and monocytic MDSCs (M-MDSCs) $\left(\mathrm{CD}_{3}{ }^{+} \mathrm{CD} 11 \mathrm{~b}^{+} \mathrm{CD} 14^{+} \mathrm{HLA}-\mathrm{DR}^{\mathrm{lo}}\right)$ could be distinguished using their phenotypic profiles (Supplemental Figure 14 and Supplemental Table 2). Patients were stratified into MDSC $^{\text {lo }}$ and MDSC $^{\text {hi }}$ cohorts (Figure 3B) for each subpopulation. Although baseline E-MDSC abundance did not correlate with improved outcomes (Supplemental Figures 15 and 16), M-MDSC ${ }^{\text {lo }}$ patients with DIPG had prolonged OS $(P=0.05, \log$-rank test $)$ relative to OS for the M-MDSC ${ }^{\text {hi }}$ cohort of patients with DIPG (Supplemental Figure 16). MDSC ${ }^{\text {lo }}$ patients with DIPG had a median OS of 13.7 months (95\% CI, 9.8-NA) compared with 9.0 months (95\% CI, 7.7-NA) for MDSC ${ }^{\text {hi }}$ patients with DIPG $(P<0.01$, log-rank test) (Figure 3C), with an estimated multivariate HR of 0.09 (95\% CI, 0.01-0.92, $P=0.04$ ) (Supplemental Table 1). Further, MDSC ${ }^{\text {hi }}$ patients had a median PFS of 2.9 months (95\% CI, 2.8-NA) compared with 5.3 months (95\% CI, 3.2-NA) for their counterparts $(P$ $=0.03$, log-rank test) (Figure 3D), with an estimated univariate HR of 3.1 (95\% CI, 1.1-8.9, P= 0.04). Analyses incorporating both strata $A$ and $B$ did not yield significant findings (Supplemental Figure 15), whereas the limited sample size of stratum B prevented a robust cohort-specific survival analysis for these patients. These associations persisted when analyzing the outcomes of patients with the top and bottom quartile of baseline MDSC abundances
(Supplemental Figure 17). However, we found that baseline MDSC frequencies did not correlate with tumor volume or age (Supplemental Figure 18). With regard to other immunoregulatory cell populations, $\mathrm{CD}^{+}$Tregs showed negligible associations with patient outcomes in the DIPG cohort (Supplemental Figure 19).

Dexamethasone administration is associated with lower rates of vaccine-specific $C D 8^{+} T$ cell responses and high MDSC levels among patients with DIPG. A total of 9 (47\%) patients with DIPG received oral dexamethasone while in the study. Six of these patients commenced dexamethasone at least 3 days before baseline PBMC collection and exhibited a trend toward higher levels of baseline circulatory MDSCs, with 4 (67\%) of the 6 patients on dexamethasone exhibiting circulatory MDSC frequencies above $5 \%$, whereas 3 (23\%) of the 13 patients not receiving dexamethasone surpassed the same threshold $(n=19)$ (Figure 4A). Among patients receiving dexamethasone who were analyzed longitudinally, 3 (50\% of 6 total) exhibited an overall decrease in H3.3K27M-reactive $\mathrm{CD}^{+} \mathrm{T}$ cells at the final collection time point relative to baseline, whereas only 1 (17\% of 6 total) showed an overall expansion (Figure 4B and Supplemental Figure 20). Furthermore, patients with DIPG treated with dexamethasone at baseline had a median OS of 9.0 months ( $n=6,95 \% \mathrm{CI}, 7.7-\mathrm{NA})$ compared with a median OS of 13.7 months $(n=13,95 \%$ CI, 9.8-NA) for untreated patients $(P<$ 0.01 , log-rank test) (Figure 4C). Although not statistically significant, patients with DIPG treated with dexamethasone had a median PFS of 3.0 months $(n=6,95 \%$ CI, 2.3-NA) compared with 5.3 $(n=13,95 \% \mathrm{CI}, 4.1-\mathrm{NA})$ for untreated patients $(P=0.09$, log-rank test) (Figure 4D). These trends persisted when combining strata A and B (Supplemental Figure 21).

Circulating tumor DNA analysis is not predictive of outcome. A cutoff threshold for positive circulating tumor DNA (ctDNA) was set at $0.001 \%$ on the basis of our previously described method (8), where sensitivity and specificity were fully described using specimens from a large cohort of patients. At baseline, we collected plasma from 27 of 29 patients for quantification of H3.3K27M mutation ( $H 3 F 3 A$ c.83A $>$ T) allelic frequency (MAF) (8-10). We detected circulating $H 3 F 3 A$ c.83A $>$ T tumor DNA H3.3K27Mspecific ctDNA in $70.4 \%$ ( $n=19$ of 27$)$ of the patients' samples, but its presence at baseline or longitudinal fluctuation in abundance did not correlate with clinical outcomes (Supplemental Figures 22 and 23) or immunological responses (unpublished observations).

Longitudinal assessment of immunological profiles in correlation with the clinical outcome of a patient with DIPG exhibiting a strong $C D 8^{+}$response to vaccination. One patient enrolled in stratum $\mathrm{A}$ demonstrated a partial radiographic response at weeks 12 and 24 (Figure 5A). Following a total of 9 vaccinations, the patient was taken off the study at week 36 because of clinical worsening but only a minor increase in tumor size, which did not meet radiographic progression criteria based on a central imaging review. The patient underwent reirradiation 10 months after the first vaccine and never received dexamethasone. The patient has not received additional therapies and remains alive 19 months after the initial diagnosis (March 2020 cutoff). A longitudinal immunological assessment revealed the time course-dependent expansion and persistence of H3.3K27M-reactive $\mathrm{CD} 8^{+} \mathrm{T}$ cells (Figure $5 \mathrm{~B})$. The continuously mounting $\mathrm{T}$ cell responses, along with the minor increase in tumor size by week 36 , suggest a possibility of an 


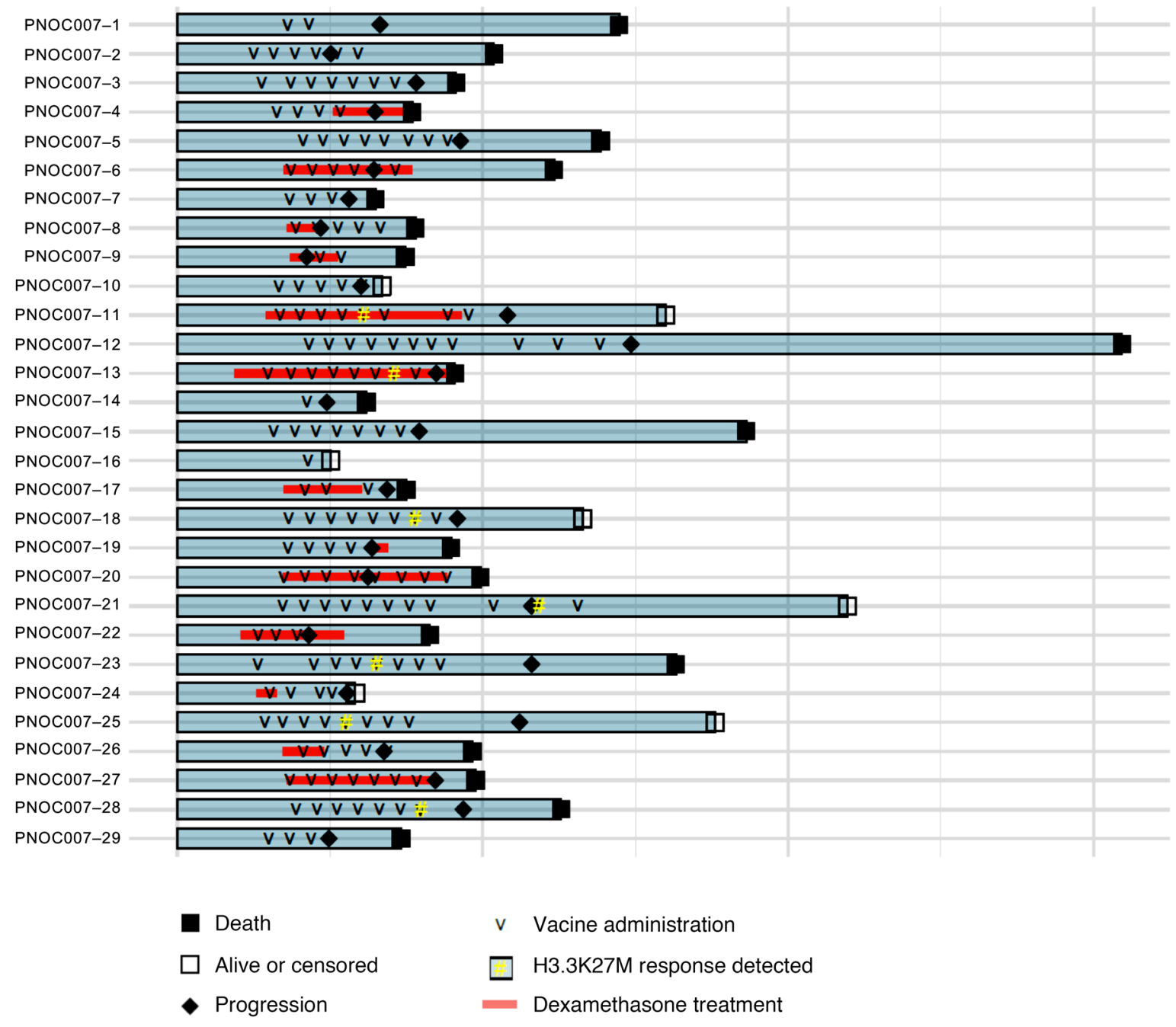

Figure 2. Individual patient study course. Depicted within this swimmer plot is the patient-specific timing of vaccine administration, dexamethasone treatment, detection of H3.3K27M-reactive T cell responses, tumor progression, and vital status (patients who withdrew consent were censored).

inflammation-associated imaging change in this case. This patient was classified as MDSC $^{\text {lo }}$ among DIPG patients with total MDSC percentages of $2.6 \%$ (median $=4.2 \%$ ), indicative of an improved prognosis among patients with DIPG, as our findings have reflected (Figure 3, C and D). Furthermore, this patient's MDSC abundance did not increase above median levels until disease progression was identified at 36 weeks (Figure 5C).

\section{Discussion}

To our knowledge, this is the first multicenter trial assessing the safety and clinical efficacy of a vaccine directed toward the novel, shared neoantigen $\mathrm{H} 3.3 \mathrm{~K}_{2} 7 \mathrm{M}_{(27-36)}$ in $\mathrm{HLA}-\mathrm{A}^{*} \mathrm{O2}: 01^{+}$ patients with $\mathrm{H} 3.3 \mathrm{~K} 27 \mathrm{M}^{+}$DIPG or DMGs. The study demonstrated safety of the regimen and supports the significance of immunological monitoring for the identification of predictive biomarkers. Moreover, clinical and immune data suggest that concurrent corticosteroid administration is associated with increased MDSC levels and lower vaccine efficacy.

The PNOC007 trial regimen demonstrated safety in regard to expected TRAEs. One child developed meningitis of unclear etiology and recovered completely with standard treatment. It remains uncertain if this event was related to the vaccine, since no additional cases were identified. The PNOC007 treatment strategy did not improve the overall outcome of $\mathrm{H} 3.3 \mathrm{~K} 27 \mathrm{M}^{+}$patients with DMG, representing the worst prognostic group among patients with DMG (11). A recent analysis from the International and European DIPG registries reported a similar OS12 of $42.3 \%$ (95\% CI, $38.1 \%-44.1 \%)(1)$.

To our knowledge, this is the first trial in which individuals with DMG were enrolled on the basis of their mutation status rather than tumor location. Outcome measures for these tumors remain a topic of ongoing prospective studies, but retrospective reviews show poor outcomes for these tumor location-specific subgroups. For example, a retrospective analysis of $\mathrm{H} 3.3 \mathrm{~K} 27 \mathrm{M}^{+} \mathrm{DMGs}$ enrolled in the German HIT-HGG registry revealed a median survival of 13 months among patients with thalamic tumors and 4.8 months among those with tumors within the spine (11), similar to our results. To date, very few peptide vaccine-based strategies have been reported for DIPGs or DMGs. We have previously reported a median survival of 12.7 months in patients with DIPG using a peptide vaccine strategy against IL-13R $\alpha$, EphA2, and survivin in combination with poly-ICLC (12). This trial used a strategy 
A
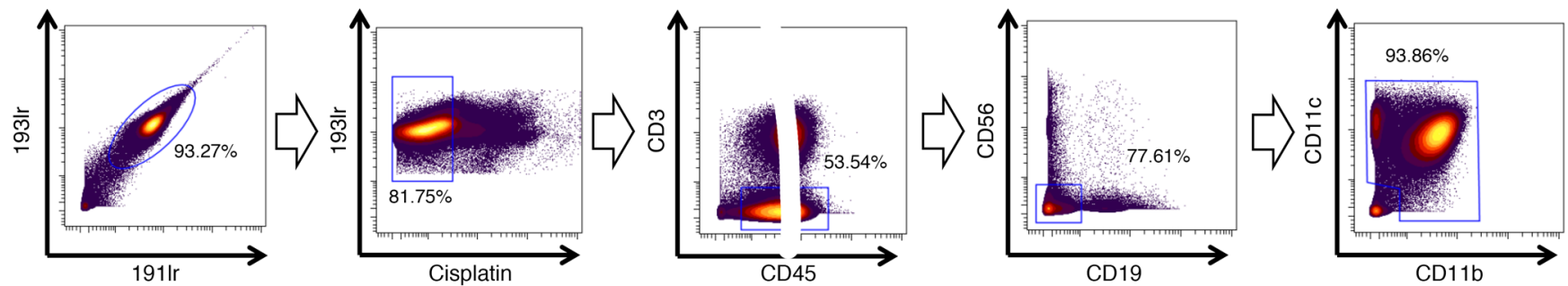

B
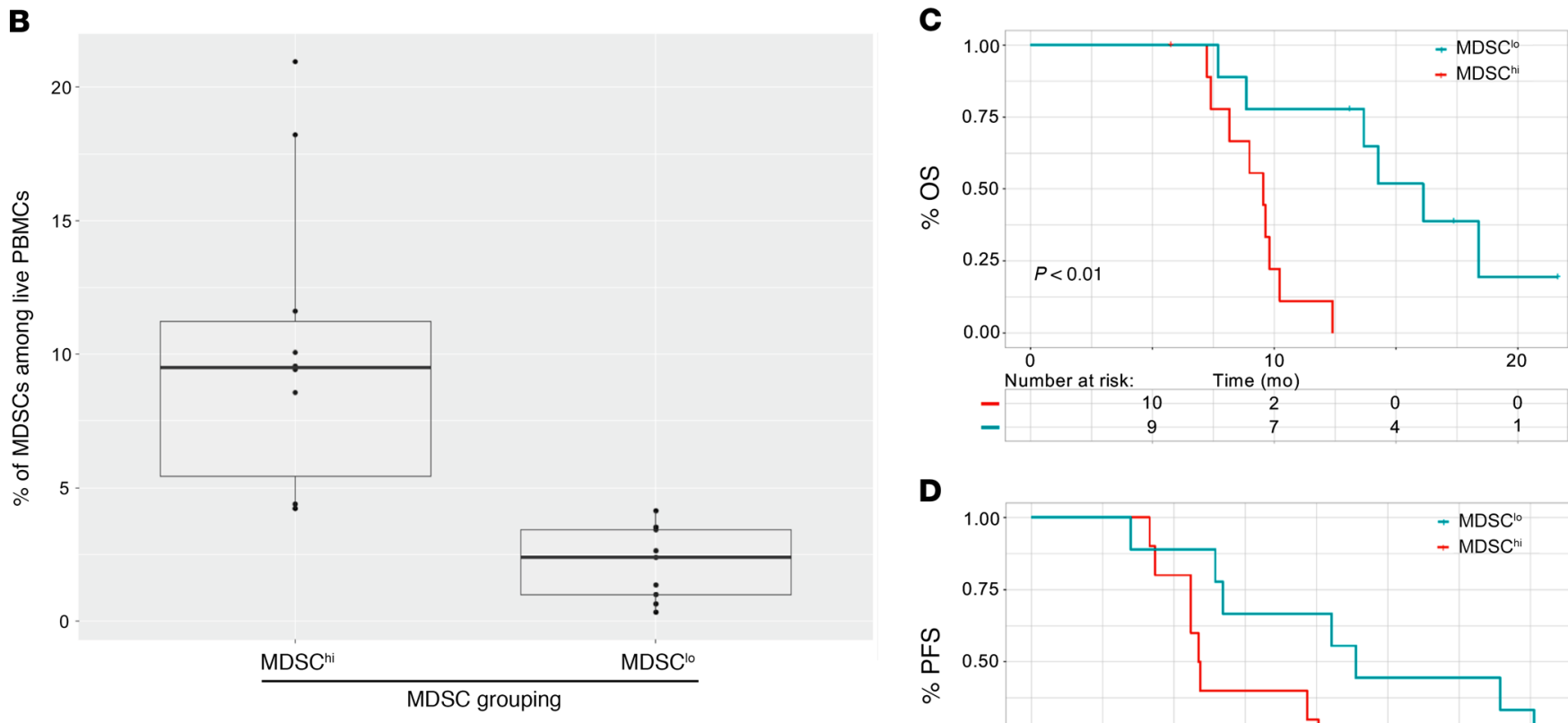

D

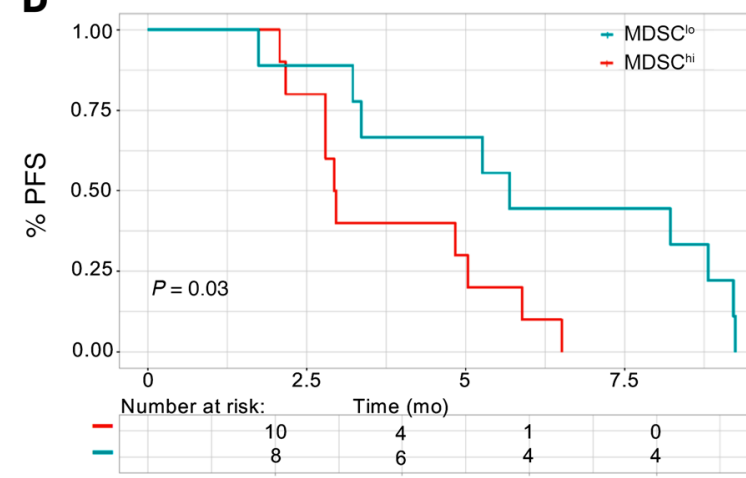

Figure 3. Mass cytometry-based analysis of circulatory MDSCs reveals an association between baseline levels of MDSCs and shortened OS and PFS. (A) Representative gating strategy to identify myeloid cells from patient-derived PBMCs by mass cytometry. Cells that stained double-positive with iridium intercalator were identified as intact cells (far left). Cells with low cisplatin staining were identified as live cells (second from left). Cells that stained negative for CD45 or positive for CD3 were excluded (middle). Cells that stained positive for CD19 or positive for CD56 were excluded (second from right). Cells that stained positive for either CD11b or CD11c were identified as myeloid cells (far right). (B) Box plot shows the range of abundance of circulatory total MDSCs among patients with DIPG classified as MDSC ${ }^{10}$ or MDSC ${ }^{\text {hi. }}$. MDSC classifications were established on the basis of a median threshold. (C and D) Kaplan-Meier survival curves contrasting (C) OS and (D) PFS of DIPG patients with baseline circulatory MDSC levels above (red) and below (blue) the median threshold, as determined by log-rank tests.

similar to that for PNOC007, however, H3.3K27M status was not available and could have influenced outcomes.

Our cytometry by time of flight-based (CyTOF-based) analyses of multiple immune subsets $(13,14)$ provided an opportunity to explore associations between expansion of H3.3K27M-reactive $\mathrm{CD}^{+} \mathrm{T}$ cell subsets and prolonged OS and PFS. Furthermore, this approach identified baseline circulatory MDSCs as a potential negative prognostic indicator for patients with DIPG. To our knowledge, this is the first CyTOF-based immunomonitoring platform used to evaluate epitope-specific $\mathrm{CD} 8^{+} \mathrm{T}$ cell responses in patients with cancer treated with peptide-based vaccines. Detection of this cell population was accomplished through HLA dextramer-based staining (15-17), as dextramers exhibit higher affinity and specificity for epitope-specific $\mathrm{CD} 8^{+} \mathrm{T}$ cell popula- tions in comparison with conventional HLA tetramers $(15,17)$. Furthermore, an increased quantity of fluorophores conjugated to each dextramer allowed for increased binding to metal-conjugated secondary antibodies, resulting in higher resolution on both fluorophore- and lanthanide-based analytical platforms. This approach maximized sensitivity on the CyTOF platform, allowing for the comparable detection of H3.3K27M-reactive CD8 ${ }^{+} \mathrm{T}$ cells between flow and mass cytometry. This study validates CyTOF as a powerful tool for high-definition immunomonitoring of $\mathrm{CD} 8^{+} \mathrm{T}$ cell-based immunotherapies.

While other immune cell populations are the focus of interest $(18,19)$ across the broad spectrum of cancer vaccines $(20)$, $\mathrm{CD}^{+} \mathrm{T}$ cells remain the primary target population for developing an efficacious antitumor immunotherapy (21). Consistent 
A

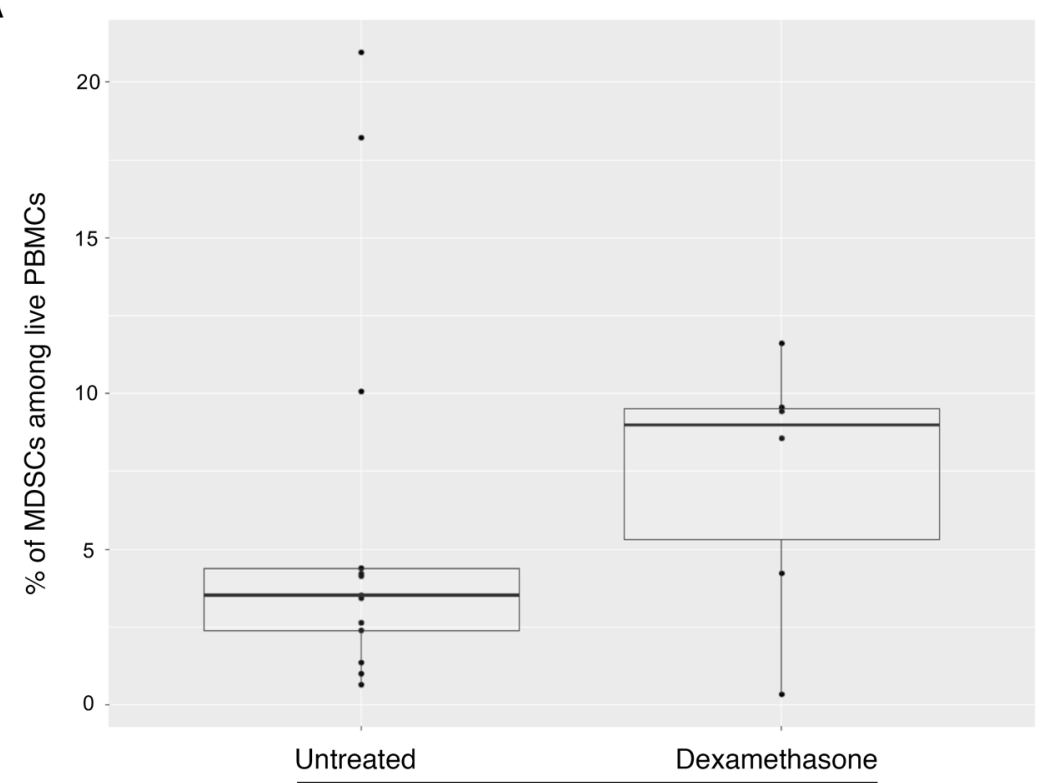

Baseline treatment group
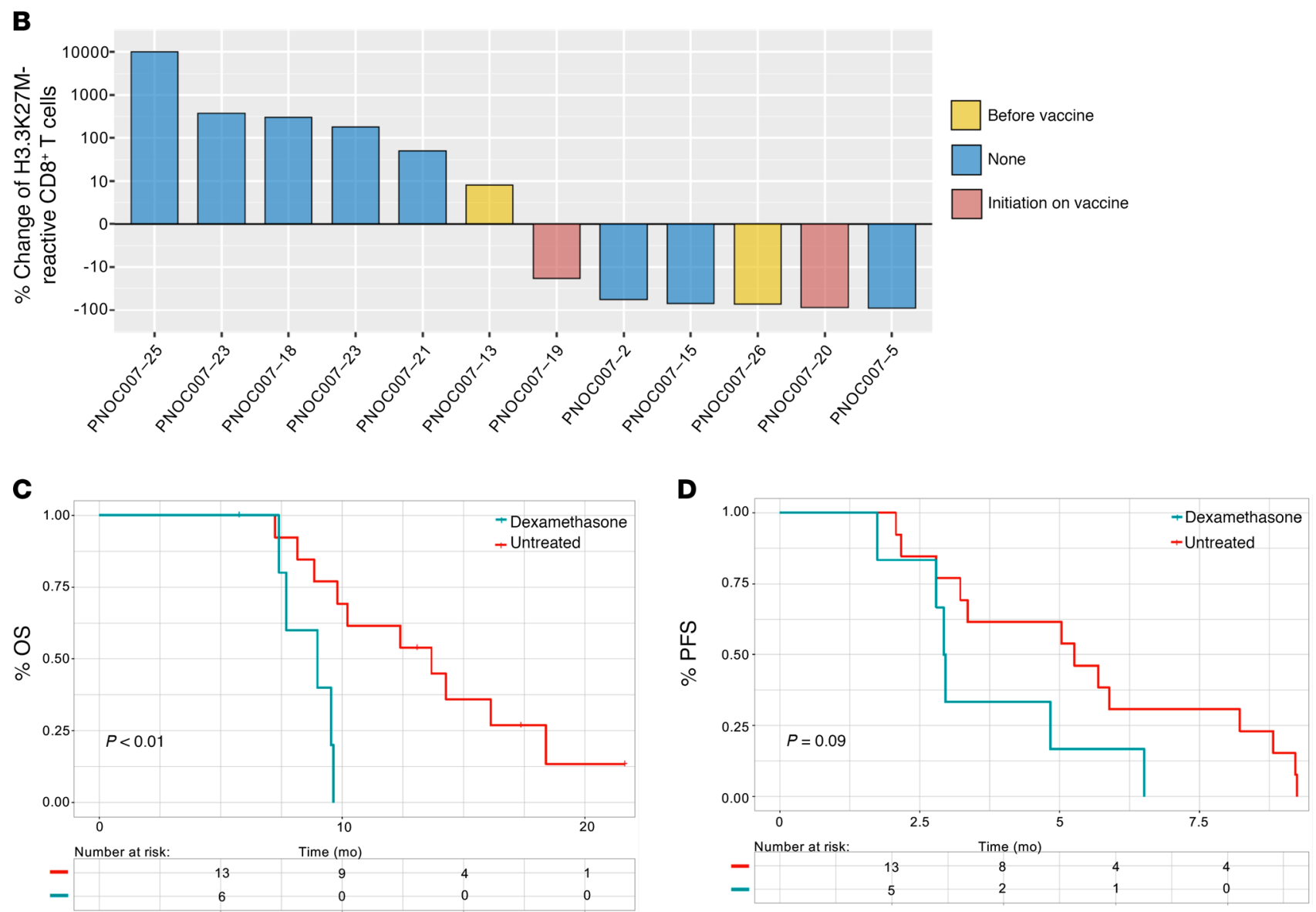

Figure 4. Administration of dexamethasone is positively associated with increased baseline MDSC levels and inversely associated with H3.3K27Mreactive CD8+ $\mathbf{T}$ cell responses and survival. (A) Box plot demonstrates the abundance of circulatory MDSCs at baseline among patients with DIPG treated with dexamethasone $(n=6)$ for at least 3 days prior to treatment relative to patients who did not receive dexamethasone $(n=13)$. (B) Waterfall plot depicts the overall longitudinal percentage change of H3.3K27M-reactive CD8 ${ }^{+} \mathrm{T}$ cells at the final time point analyzed relative to baseline for each patient with DIPG who was treated with dexamethasone. Yellow bars indicate the pre-treatment period; red bars indicate patients who received dexamethasone after commencement of vaccination; and blue bars indicate patients who did not receive dexamethasone while in the study. (C and $\mathbf{D})$ Kaplan-Meier survival curves contrasting the (C) OS and (D) PFS of patients with DIPG based on pretreatment dexamethasone administration, as determined by log-rank tests. 
with this paradigm, the expansion of $\mathrm{H} 3.3 \mathrm{~K} 27 \mathrm{M}-$ reactive $\mathrm{CD}^{+}$ $\mathrm{T}$ cells, but not bulk CD8 ${ }^{+} \mathrm{T}$ cells, is suggestive of prolonged OS. Although this expansion provides insight into patient-specific immunological outcomes, it does not account for phenotypic composition or functionality. As peripheral Tex expansion has been correlated with poor prognoses in several cancer types $(22,23)$, the discrimination of this cell population, among others, is necessary for a contextual assessment of immunological responses. Our data demonstrated no association between Tex expansion and favorable outcomes for patients. However, the expansion of H3.3K27M-reactive Tem cell subpopulations, which exhibit cytolytic and tissue localization capabilities (24), was associated with clinical benefits among patients with DMG, consistent with previous reports (25-27).

One could raise a concern about whether the observed H3.3K27M-reactive $\mathrm{CD}^{+} \mathrm{T}$ cell responses merely reflect overall immune competency, which is often compromised in these patients. Our multivariate analysis on bulk $\mathrm{CD} 8^{+} \mathrm{T}$ cells suggested that this was not the case. Another line of concern is the possibility that patients with intrinsically slow-growing tumors may have received more vaccines than those with fast-growing tumors, thereby mounting higher levels of vaccine-reactive responses. Based on our analyses, this does not appear to be the case, as the median numbers of vaccines preceding immunological responses among responders was the same as the median number of total vaccines among nonresponders.

To date, there are no vaccine regimens that have been shown to be efficacious in CNS malignancies, as shown by randomized studies with adequate statistical powers. As we discussed in our recent review articles $(28,29)$, we recognize that underlying these failures are a number of mechanistic challenges, such as antigen-heterogeneity, local and systemic immunosuppression, and lack of adequate homing of effector immune cells. Although most tumor-specific antigens are expressed heterogeneously within the tumor (29), H3.3K27M appears to be uniformly expressed in the tumor tissue (7).

In terms of relevant immunosuppressive mechanisms, MDSCs have attracted particular interest not only as tumor promoters (30) but as entities antagonistic to peptide vaccine efficacy (31-33) in preclinical models. Our data reflected this, as MDSC ${ }^{\text {lo }}$ DIPG patients exhibited improved outcomes relative to their counterparts. It is also noteworthy that our data set showed an absence of associations between relative circulatory MDSC abundance and both the patient's age and tumor volume. Although Tregs are important immunosuppressive cells, our analyses did not detect any association between Tregs and patient outcomes in the DIPG cohort, corroborating recent high-throughput analyses of PBMC samples in patients with glioblastoma (30). However, these results warrant caution. As cryopreservation and paraformaldehyde fixation are known to reduce polymorphonuclear MDSC viability (34), E-MDSCs and M-MDSCs were used as proxies for total MDSC quantification. Our data did not establish a robust correlation between baseline MDSC levels and longitudinal H3.3K27Mreactive $C D 8^{+} \mathrm{T}$ cell frequencies, which may have been affected by the limited longitudinal samples available. Nevertheless, MDSCs represent an enticing target for combinatorial therapies (35) that maximize vaccine efficacy.
Corticosteroids are often administered to patients with DIPG or DMG to treat neurological dysfunction caused by tumor- or treatment-related edema. However, steroid dependency has been associated with a worse prognosis (36) and may negatively affect responses to immunotherapies (5). Consistent with recent preclinical in vitro and in vivo studies $(37,38)$, our data suggest a negative association between dexamethasone administration and the longitudinal expansion of vaccine-reactive $\mathrm{CD} 8^{+} \mathrm{T}$ cells, consistent with a recent report (5). It is conceivable that tumor size and growth rate, which typically necessitate the use of corticosteroids, may confound our observations. Our data suggest a lack of association between the baseline tumor size and outcomes (Supplemental Figures 24 and 25), consistent with a recent study involving molecularly characterized DIPGs (39). However, given the small sample size, we cannot fully exclude that dexamethasone use, independent of its impact on vaccine efficacy, leads to worse outcomes. Nonetheless, this association calls for additional corroborative studies.

Key limitations of our study include the relatively small number of patients and longitudinal samples for immune phenotypic analyses and classification of immunological responses. Some study participants were lost to follow-up because of clinical worsening of their disease before post-baseline PBMC collection, limiting the availability of longitudinal samples to $62 \%$ (18 of 29) of the patients enrolled. Systematic evaluation of pre- and post-treatment tumor microenvironment samples would have offered more insights on the vaccine's effects, such as $\mathrm{T}$ cell infiltration as well as possible antigen loss and HLA downregulation. To date, post-treatment biopsy of DMGs remains a controversial topic in pediatric neuro-oncology $(10,40)$, and this limitation extended to our current study. At the time of submission, there were too few data to establish corroborations with CyTOF analyses (Supplemental Figures 26 and 27). Given the clinical information gained by pathologic assessment of post-treatment tumor biopsies, these should be considered in future trials to better understand the effect of our therapies.

In conclusion, our data reflect the safety of the regimen and highlight the encouraging roles of $\mathrm{H} 3.3 \mathrm{~K} 27 \mathrm{M}$-specific $\mathrm{CD}^{+} \mathrm{T}$ cell responses in these patients, warranting further studies targeting the H3.3K27M epitope. Such studies should focus on a vaccine regimen with anti-PD-1 therapy (based on expression of PD-1 on H3.3K27M-reactive CD8 ${ }^{+} \mathrm{T}$ cells) and adoptive transfer of $\mathrm{T}$ cells transduced with the H3.3K27M-reactive T cell receptor (4).

\section{Methods}

Study design. PNOC007 (ClinicalTrials.gov ID: NCT02960230) is a pilot trial assessing the safety, immunoreactivity, and efficacy of subcutaneous administration of the H3.3K27M synthetic peptide combined with helper TT peptide (Tet ${ }_{\text {A830 }}$, PolyPeptide Group) $(41,42)$ emulsified in Montanide-ISA 51 VG (SEPPIC) and in combination with poly-ICLC (Hiltonol, Oncovir) in patients 3 to 21 years of age with newly diagnosed DIPG (stratum A) or nonpontine DMG, including spinal cord DMGs (stratum B). Key inclusion criteria were the confirmation of

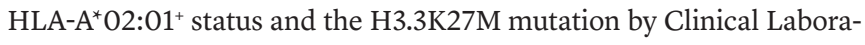
tory Improvement Amendments-approved (CLIA-approved) sequencing of tumor tissue as well as adequate organ function. Exclusion criteria included known immune system disorders and treatment with other 
A
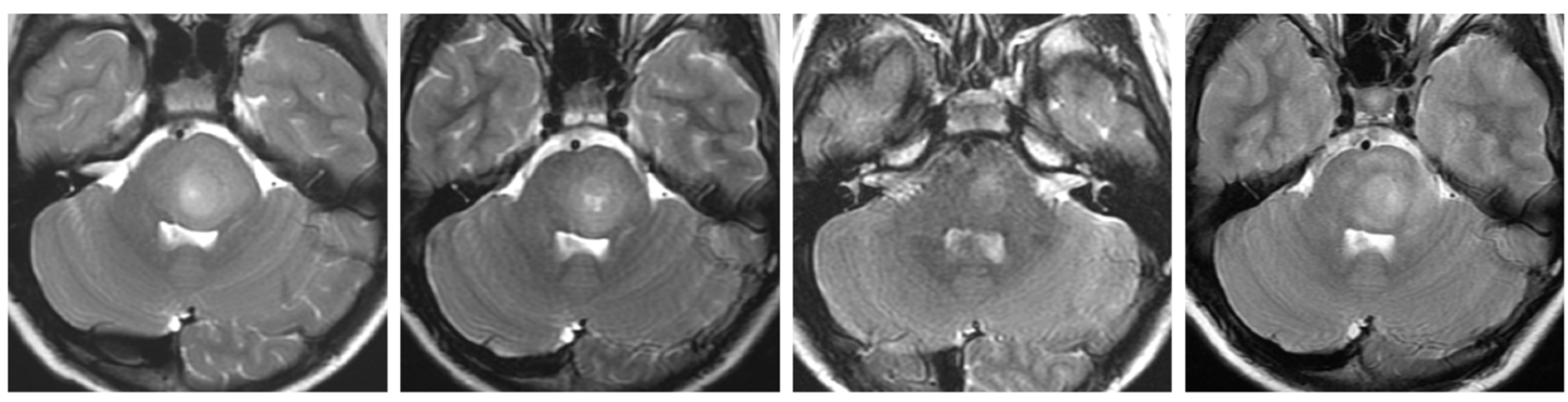

B

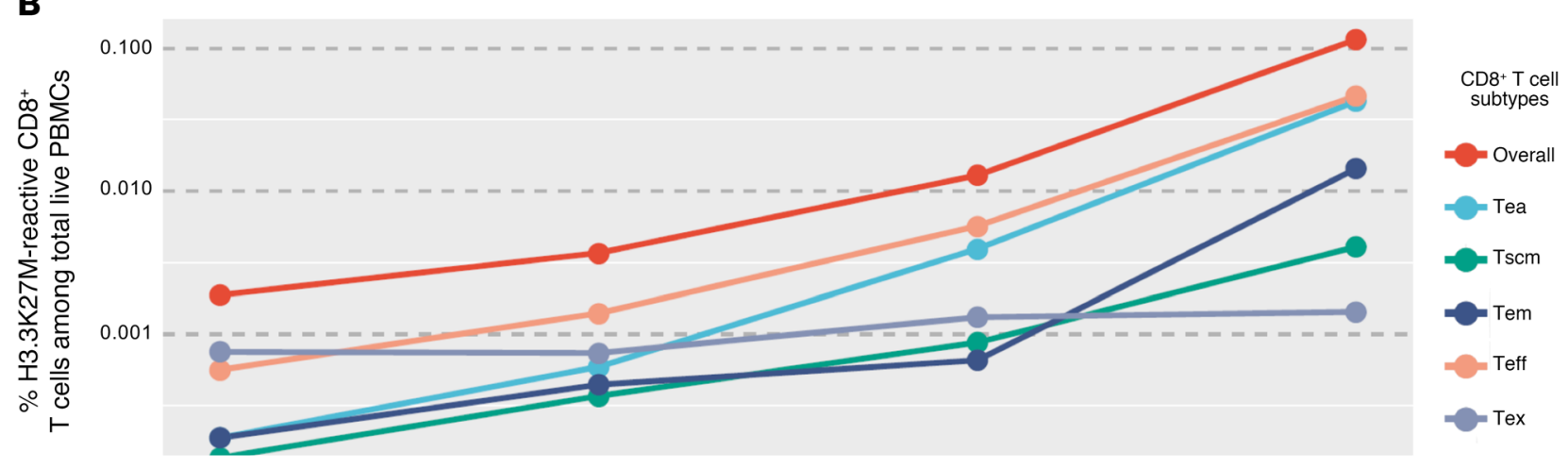

C

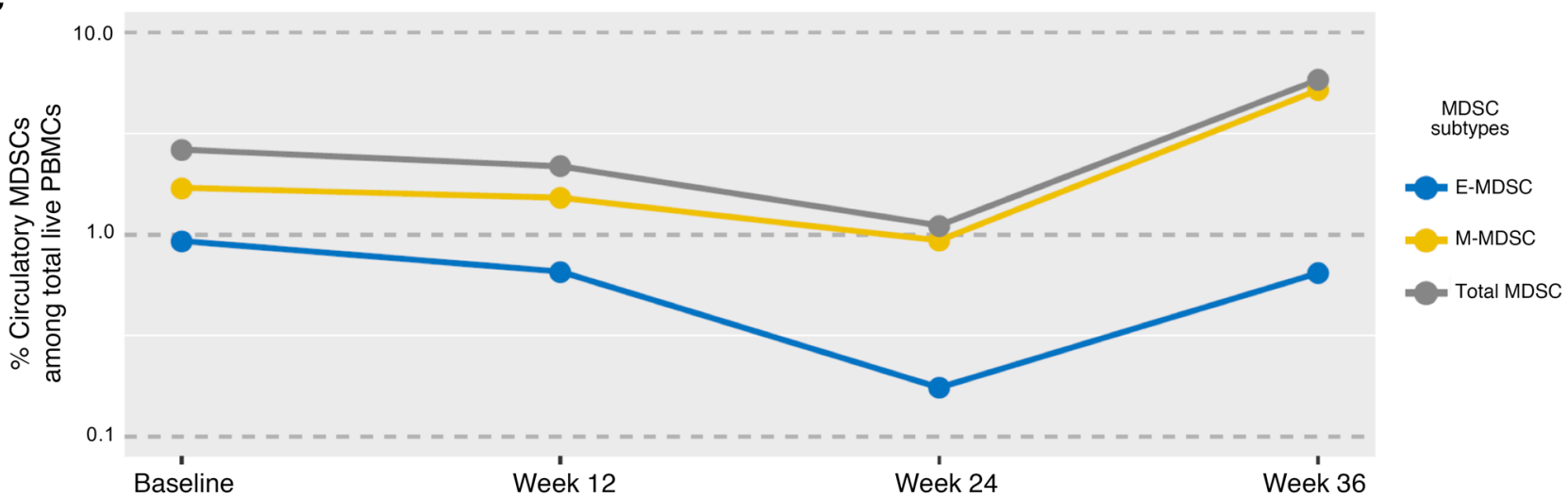

Figure 5. A patient who demonstrated a continued expansion of H3.3K27M-reactive CD8 ${ }^{+}$T cells. (A) MR images of a patient at baseline (left), week 12 (second from left), week 24 (second from right), and week 36 (far right). The patient (PNOC007-25) was found to have local progression in week 36. (B) Plot shows the longitudinal percentage of H3.3K27M-reactive CD8 ${ }^{+} \mathrm{T}$ cell subpopulations among live PBMCs. (C) Plot depicts the longitudinal percentage of circulatory MDSC subsets among live PBMCs.

investigational or anticancer agents with the exception of temozolomide (maximum $90 \mathrm{mg} / \mathrm{m}^{2} /$ dose) during focal RT. Patients must have been either off systemic steroids or have been on a stable dose of dexamethasone not exceeding $0.1 \mathrm{mg} / \mathrm{kg} /$ day (maximum $4 \mathrm{mg} /$ day). All patients were enrolled upon completion of focal RT.

Participating PNOC sites received the H3.3K27M synthetic peptide vaccine combined with a TT peptide through a central supply from the Immunologic Monitoring and Cellular Products Laboratory at the University (IMCPL) of Pittsburgh. Pharmacists at each site were trained for the emulsification process and prepared each vaccine emulsion $(300 \mu \mathrm{g} / \mathrm{dose} \mathrm{H} 3.3 \mathrm{~K} 27 \mathrm{M}$ synthetic peptide and $200 \mu \mathrm{g} / \mathrm{dose}$ TT peptide in Montanide). The vaccine was administered subcutaneously in either the arm or thigh, beginning 2-8 weeks after completion of RT, and given every 3 weeks for the first 8 doses followed by every 6 weeks (Supplemental Figure 28) on an outpatient basis for a maximum treatment period of 96 weeks. Vaccine was administered at the same location unless prevented by an adverse skin reaction. Concurrently, the adjuvant poly-ICLC was administered $(30 \mu \mathrm{g} / \mathrm{kg})$ intramuscularly within $2 \mathrm{~cm}$ of the vaccine injection site $(43,44)$. Follow-up data were collected through February 2020.

Study assessments. Patients were monitored by laboratory assessments and physical examination. Tumor evaluations were performed with MRI at baseline and every 12 weeks. Blood was collected to assess ctDNA at baseline and every 12 weeks (10). PBMCs were collected at baseline and at weeks 12,18 , and 24, and then every 12 weeks thereafter for CyTOF-based immunomonitoring assessments. 
Central imaging review. A central radiology review of all cases was conducted retrospectively using response assessment in neuro-oncology (RANO) (45) criteria. MRI with T1 (with and without contrast enhancement), T2, and T2 fluid-attenuated inversion recovery (FLAIR) was used to measure tumor size. Anterior-posterior and transverse dimensions were measured using the Horos (version 3.0) software package. Progressive disease on MRI was defined as a greater than $25 \%$ increase in the sum of perpendicular diameters and/or development of new enhancing or nonenhancing lesions by a board-certified neuroradiologist.

Study endpoints. The study's primary endpoints were the evaluation of safety for all patients and OS at 12 months for patients enrolled in stratum A. The exploratory endpoints included CyTOFbased evaluation of $\mathrm{H} 3.3 \mathrm{~K} 27 \mathrm{M}$-reactive $\mathrm{CD}^{+} \mathrm{T}$ cell responses in PBMC samples, immunohistochemical analysis of available tumor tissue, and ctDNA analyses.

Safety. Adverse events (AEs), serious adverse events (SAEs), and RLTs were reported from vaccine initiation out to 30 days after the last study treatment. Grading was based on Common Terminology Criteria for Adverse Events (CTCAE), version 4.0 (46). RLTs were defined as any grade- 2 or greater autoimmune reaction; any grade- 3 or greater hematologic or nonhematologic toxicity with the exception of lymphopenia; any life-threatening event; and any other unexpected grade-2 or greater neurological deficit that was related to the vaccine therapy and did not respond to a trial of dexamethasone or bevacizumab. AEs, SAEs, and RLTs were reviewed weekly by the study chairs and PNOC leadership according to PNOC standardized operating procedures for safety monitoring. The data monitoring and safety committee (DSMC) at UCSF monitored the trial.

An early stopping rule was implemented on the basis of RLT incidences as follows: the first 3 patients in each stratum were observed for at least 12 weeks before the next set of patients were enrolled. If a RLT occurred in 1 of the first 3 patients, an additional 3 patients would be enrolled in that stratum and observed for at least 12 weeks. If fewer than 2 of the first 6 patients in each stratum experienced a RLT within at least 12 weeks of follow-up, the remaining patients would be enrolled. If a RLT occurred in 2 or more of the first 6 patients, accrual would be suspended. Therefore, with 6 patients enrolled, there was an $88 \%$ probability of detecting 1 or more RLTs with an underlying rate of $30 \%$. There was a 74\% probability of detecting 1 or more RLTs with an underlying rate of $20 \%$. In stratum A, if all 19 patients were enrolled, there was an $86 \%$ probability of detecting 1 or more RLTs with an underlying rate of $10 \%$ and a $62 \%$ probability with an underlying rate of $5 \%$.

Mass cytometric data processing. Data acquisition for $\mathrm{H} 3.3 \mathrm{~K} 27 \mathrm{M}$ epitope-specific $\mathrm{CD}^{+} \mathrm{T}$ cell responses was conducted using the Fluidigm Helios CyTOF system at the UCSF Parnassus Flow Cytometry Core. The sensitivity and specificity of the HLA-A ${ }^{*} 02: 01-\mathrm{H} 3.3 \mathrm{~K} 27$ dextramer (Immudex) were validated by positive staining of $\mathrm{CD}^{+}$ $\mathrm{T}$ cells transduced with an $\mathrm{H} 3.3 \mathrm{~K} 27 \mathrm{M}$-specific $\mathrm{T}$ cell receptor (4) and negligible staining in nontransduced $\mathrm{CD} 8^{+} \mathrm{T}$ cells, respectively. H3.3K27M-reactive CD8 ${ }^{+} \mathrm{T}$ cells were pooled from all patients and displayed via a t-SNE plot in the cytofkit package (47).

Statistics for OS and immune responses. For stratum A, with a null hypothesis that OS12 is $40 \%$, accrual of 19 patients provided $80 \%$ power with a target overall type I error of 0.05 to detect a $30 \%$ difference in OS12 using a 1-sided exact binomial test. A null OS12 rate of $40 \%$ was chosen on the basis of prior studies $\left(48^{-50)}\right.$ and review of outcomes of DIPG patients. Time-to-event analyses were calculated using the Kaplan-Meier method from the date of diagnosis to the date of censoring or death. Patients who withdrew consent were lost to follow-up or were still alive at the study cutoff date were censored for OS-based survival analyses. The log-rank and likelihood ratio tests were used for comparisons between groups in Kaplan-Meier and Cox proportional HR analyses, respectively. OS was used as the primary survival parameter for the Cox proportional HR multivariate analyses, which were conducted using nonconfounding variables $(P>0.10)$ as determined by Fisher's exact test. A $P$ value of less than 0.05 was considered statistically significant.

For stratum B, pilot OS data were obtained in this specific subpopulation to support the design of the subsequent phase 2 trial $(n=10)$.

An overall immunological response to the vaccine was defined as at least a $25 \%$ post-vaccination increase in the proportion of H3.3K27M-reactive CD8 ${ }^{+} \mathrm{T}$ cells among total live PBMCs relative to baseline. An analogous approach was used for classifying the expansion of tSNE-stratified bulk and H3.3K27M-reactive CD8 ${ }^{+} \mathrm{T}$ cell subpopulations.

Patients were further classified by relative abundance of PBMC-derived MDSC subsets at baseline. Myeloid cells from all patients were pooled and stratified on a tSNE plot, revealing 32 discrete clusters (Supplemental Figure 14), of which E-MDSC and M-MDSC subsets (51) were quantified. Patients were assigned

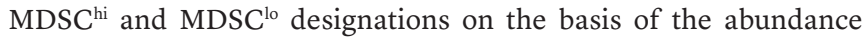
of these cell populations relative to median levels at baseline. This approach was extrapolated to incorporate total (sum of E-MDSCs and M-MDSCs) MDSC levels.

Data analyses and visualization were performed using the ggplot2 (52) and cytofkit (47) R packages.

Study approval. Prior to trial activation, the appropriate approvals were obtained from the relevant regulatory agencies, including the FDA, for use of the investigational new drug (IND no. 17070), as well as IRB approval from UCSF, Lurie Children's Hospital of Chicago, Seattle Children's Hospital (Seattle, Washington, USA), Oregon Health \& Science University, UCSD and Rady Children's Hospital, Dana-Farber Cancer Institute, Children's National Medical Center (Washington, DC, USA), Washington University in St. Louis, The Children's Hospital Of Philadelphia (Philadelphia, Pennsylvania, USA), St. Jude Children's Research Hospital (Memphis, Tennessee, USA), Nationwide Children's (Columbus, Ohio, USA), Texas Children's Hospital (Houston, Texas, USA), Primary Children's Hospital (Salt Lake City, Utah, USA), and Children's Minnesota (Minneapolis, Minnesota, USA). All study participants or their parents provided informed consent and age-appropriate assent according to institutional IRB guidelines.

\section{Author contributions}

SM, MDP, and HO conceptualized and designed the study. JMT, TN, PBW, NDA, KO, LHB, and RDG analyzed PBMC and tumor tissue samples. JEVM conducted imaging data analyses. ERB and JN evaluated ctDNA. SM, RRL, SG, AB, SNC, NSW, JRC, KG, and KJN enrolled and managed the patients. AMS provided poly-ICLC. AMM supervised the statistical and analytical methods used. All authors participated in writing and revising the manuscript. SM and HO, the study chairs, and SM and MP, on behalf of the PNOC leadership, reviewed AEs, SAEs, and RLTs weekly per PNOC standardized operating procedures for safety monitoring. 


\section{Acknowledgments}

The authors thank the following individuals and organizations: the study participants and their families; all PNOC staff for their assistance; all PNOC principal investigators supporting the study; members of the IMCPL at the University of Pittsburgh for preparation and distribution of the vaccine (supported in part by award P30 CA047904); the UCSF Parnassus Flow Cytometry Core for mass cytometry-related services (P30 DK063720) and use of the CyTOF2 "Charmander" (S10 1S10OD018040-01); the USCF data safety monitoring committee; Lawrence Fong and the Cancer Immunotherapy Laboratory (CIL) at UCSF for the processing and banking of patient-derived peripheral blood samples; Maryam Shahin for contributions to the optimization of the CyTOF analytical platform; Marie-Eve Koziol of SEPPIC Inc. for training research nurses in the preparation of Montanide emulsions; Nich- olas Bayless and Pier Federico Gherardini at the Parker Institute for Cancer Immunotherapy (PICI) for computational analyses of the mass cytometric data; and the Ian's Friends Foundation.

Prior publication: This work was partially presented at the 5th Biennial Conference on Pediatric Neuro-Oncology Basic and Translational Research (May 4, 2019, San Francisco, California, USA), as well as the 24th Annual Meeting and Education Day of the Society for Neuro-Oncology (November 24, 2019, Phoenix, Arizona, USA).

Address correspondence to: Hideho Okada, Neurological Surgery, Helen Diller Family Cancer Research Building, UCSF, HD 472, 1450 3rd Street, San Francisco, California 94158, USA. Phone: 415.476.1637; Email: hideho.okada@ucsf.edu.
1. Hoffman LM, et al. Clinical, radiologic, pathologic, and molecular characteristics of longterm survivors of diffuse intrinsic pontine glioma (DIPG): a collaborative report from the International and European Society for Pediatric Oncology DIPG Registries. JClin Oncol. 2018;36(19):1963-1972.

2. Schwartzentruber J, et al. Driver mutations in histone $\mathrm{H} 3.3$ and chromatin remodelling genes in paediatric glioblastoma. Nature. 2012;482(7384):226-231.

3. Wu G, et al. Somatic histone $\mathrm{H} 3$ alterations in pediatric diffuse intrinsic pontine gliomas and non-brainstem glioblastomas. Nat Genet. 2012;44(3):251-253.

4. Chheda ZS, et al. Novel and shared neoantigen derived from histone 3 variant $\mathrm{H} 3.3 \mathrm{~K} 27 \mathrm{M}$ mutation for glioma $\mathrm{T}$ cell therapy. JExp Med. 2018;215(1):141-157.

5. Keskin DB, et al. Neoantigen vaccine generates intratumoral T cell responses in phase Ib glioblastoma trial. Nature. 2019;565(7738):234-239.

6. Khuong-Quang DA, et al. K27M mutation in histone H3.3 defines clinically and biologically distinct subgroups of pediatric diffuse intrinsic pontine gliomas. Acta Neuropathol. 2012;124(3):439-447.

7. Solomon DA, et al. Diffuse midline gliomas with histone H3-K27M mutation: a series of 47 cases assessing the spectrum of morphologic variation and associated genetic alterations. Brain Pathol. 2016;26(5):569-580.

8. Bonner ER, et al. Detection and monitoring of tumor associated circulating DNA in patient biofluids. JVis Exp. 2019;(148):e59721.

9. Panditharatna E, et al. Clinically relevant and minimally invasive tumor surveillance of pediatric diffuse midline gliomas using patient-derived liquid biopsy. Clin Cancer Res. 2018;24(23):5850-5859.

10. Mueller S, et al. A pilot precision medicine trial for children with diffuse intrinsic pontine glioma-PNOC003: a report from the Pacific Pediatric Neuro-Oncology Consortium. Int J Cancer. 2019;145(7):1889-1901.

11. Karremann M, et al. Diffuse high-grade gliomas with H3 K27M mutations carry a dismal prognosis independent of tumor location. Neurooncology. 2018;20(1):123-131.
12. Pollack IF, et al. Antigen-specific immune responses and clinical outcome after vaccination with glioma-associated antigen peptides and polyinosinic-polycytidylic acid stabilized by lysine and carboxymethylcellulose in children with newly diagnosed malignant brainstem and nonbrainstem gliomas. JClin Oncol. 2014;32(19):2050-2058.

13. Ornatsky O, Bandura D, Baranov V, Nitz M, Winnik MA, Tanner S. Highly multiparametric analysis by mass cytometry. J Immunol Methods. 2010;361(1-2):1-20.

14. Saeys Y, Van Gassen S, Lambrecht BN. Computational flow cytometry: helping to make sense of high-dimensional immunology data. Nat Rev Immunol. 2016;16(7):449-462.

15. Batard P, et al. Dextramers: new generation of fluorescent MHC class I/peptide multimers for visualization of antigen-specific $\mathrm{CD}^{+} \mathrm{T}$ cells. J Immunol Methods. 2006;310(1-2):136-148.

16. Dolton $\mathrm{G}$, et al. Comparison of peptide-major histocompatibility complex tetramers and dextramers for the identification of antigen-specific T cells. Clin Exp Immunol. 2014;177(1):47-63.

17. Tario JD, et al. Dextramer reagents are effective tools for quantifying CMV antigen-specific $\mathrm{T}$ cells from peripheral blood samples. Cytometry $B$ Clin Cytom. 2015;88(1):6-20.

18. Mastelic-Gavillet B, Balint K, Boudousquie C, Gannon PO, Kandalaft LE. Personalized dendritic cell vaccines -- recent breakthroughs and encouraging clinical results. Front Immunol. 2019;10:766.

19. Shimasaki N, Jain A, Campana D. NK cells for cancer immunotherapy. Nat Rev Drug Discov. 2020;19(3):200-218.

20. Sahin U, Türeci Ö. Personalized vaccines for cancer immunotherapy. Science. 2018;359(6382):1355-1360.

21. Durgeau A, Virk Y, Corgnac S, Mami-Chouaib F. Recent advances in targeting CD8 T-cell immunity for more effective cancer immunotherapy. Front Immunol. 2018;9:14

22. Kamphorst AO, et al. Proliferation of PD $-1^{+}$CD 8 $T$ cells in peripheral blood after PD-1-targeted therapy in lung cancer patients. Proc Natl Acad Sci USA. 2017;114(19):4993-4998.

23. Ma J, et al. PD $1^{\mathrm{Hi}} \mathrm{CD} 8^{+} \mathrm{T}$ cells correlate with exhausted signature and poor clinical outcome in hepatocellular carcinoma. JImmunother Cancer. 2019;7(1):331.

24. Sallusto F, Lenig D, Förster R, Lipp M, Lanzavecchia A. Two subsets of memory T lymphocytes with distinct homing potentials and effector functions. Nature. 1999;401(6754):708-712.

25. van Duikeren $S$, et al. Vaccine-induced effectormemory $\mathrm{CD}^{+} \mathrm{T}$ cell responses predict therapeutic efficacy against tumors. Jimmunol. 2012;189(7):3397-3403.

26. Lieber S, et al. Prognosis of ovarian cancer is associated with effector memory $\mathrm{CD}^{+} \mathrm{T}$ cell accumulation in ascites, CXCL9 levels and activation-triggered signal transduction in $\mathrm{T}$ cells. Oncoimmunology. 2018;7(5):e1424672.

27. Wistuba-Hamprecht K, et al. Peripheral CD8 effector-memory type $1 \mathrm{~T}$-cells correlate with outcome in ipilimumab-treated stage IV melanoma patients. Eur J Cancer. 2017;73:61-70.

28. Kwok D, Okada H. T-Cell based therapies for overcoming neuroanatomical and immunosuppressive challenges within the glioma microenvironment. J Neurooncol. 2020;147(2):281-295.

29. Nejo T, Yamamichi A, Almeida ND, Goretsky YE, Okada H. Tumor antigens in glioma. Semin Immunol. 2020;47:101385.

30. Alban TJ, et al. Global immune fingerprinting in glioblastoma patient peripheral blood reveals immune-suppression signatures associated with prognosis. JCI Insight. 2018;3(21):122264.

31. Weiss T, Vitacolonna M, Zöller M. The efficacy of an IL-1alpha vaccine depends on IL-1RI availability and concomitant myeloid-derived suppressor cell reduction. J Immunother. 2009;32(6):552-564.

32. Lee JM, Seo JH, Kim YJ, Kim YS, Ko HJ, Kang CY. The restoration of myeloid-derived suppressor cells as functional antigen-presenting cells by NKT cell help and all-trans-retinoic acid treatment. Int JCancer. 2012;131(3):741-751.

33. Song $\mathrm{X}$, et al. A tritherapy combination of a fusion protein vaccine with immune-modulating doses of sequential chemotherapies in an optimized regimen completely eradicates large tumors in mice. Int J Cancer. 2011;128(5):1129-1138.

34. Kotsakis A, Harasymczuk M, Schilling B, Georgoulias V, Argiris A, Whiteside TL. Myeloid- 
derived suppressor cell measurements in fresh and cryopreserved blood samples. JImmunol Methods. 2012;381(1-2):14-22.

35. Albeituni SH, Ding C, Yan J. Hampering immune suppressors: therapeutic targeting of myeloidderived suppressor cells in cancer. Cancer J. 2013;19(6):490-501.

36. Lobon-Iglesias MJ, et al. Diffuse intrinsic pontine gliomas (DIPG) at recurrence: is there a window to test new therapies in some patients? J Neurooncol. 2018;137(1):111-118.

37. Yang F, Li Y, Zhang Q, Tan L, Peng L, Zhao Y. The effect of immunosuppressive drugs on MDSCs in transplantation. JImmunol Res. 2018;2018:5414808.

38. Zhao Y, et al. Dexamethasone-induced myeloid-derived suppressor cells prolong allo cardiac graft survival through iNOS- and glucocorticoid receptor-dependent mechanism. Front Immunol. 2018;9:282.

39. Jaimes $C$, et al. MR imaging correlates for molecular and mutational analyses in children with diffuse intrinsic pontine glioma. AJNR Am J Neuroradiol. 2020;41(5):874-881.

40. Gupta N, et al. Prospective feasibility and safety assessment of surgical biopsy for patients with newly diagnosed diffuse intrinsic pontine glioma.
Neuro-oncology. 2018;20(11):1547-1555.

41. Slingluff CL, et al. Phase I trial of a melanoma vaccine with gp100(280-288) peptide and tetanus helper peptide in adjuvant: immunologic and clinical outcomes. Clin Cancer Res. 2001;7(10):3012-3024.

42. Okada $\mathrm{H}$, et al. Induction of robust type-I $\mathrm{CD} 8^{+}$ T-cell responses in WHO grade 2 low-grade glioma patients receiving peptide-based vaccines in combination with poly-ICLC. Clin Cancer Res. 2015;21(2):286-294.

43. Zhu X, et al. Poly-ICLC promotes the infiltration of effector $\mathrm{T}$ cells into intracranial gliomas via induction of CXCL1O in IFN-alpha and IFN-gamma dependent manners. Cancer Immunol Immunother. 2010;59(9):1401-1409.

44. Zhu X, et al. Toll like receptor-3 ligand poly-ICLC promotes the efficacy of peripheral vaccinations with tumor antigen-derived peptide epitopes in murine CNS tumor models. J Transl Med. 2007;5:10.

45. Wen PY, et al. Updated response assessment criteria for high-grade gliomas: response assessment in neuro-oncology working group. JClin Oncol. 2010;28(11):1963-1972.

46. Chen AP, et al. Grading dermatologic adverse events of cancer treatments: the common termi- nology criteria for adverse events version 4.0. J Am Acad Dermatol. 2012;67(5):1025-1039.

47. Chen H, Lau MC, Wong MT, Newell EW, Poidinger M, Chen J. Cytofkit: a Bioconductor package for an Integrated Mass cytometry data analysis pipeline. PLoS Comput Biol. 2016;12(9):e1005112.

48. Bailey S, et al. Diffuse intrinsic pontine glioma treated with prolonged temozolomide and radiotherapy--results of a United Kingdom phase II trial (CNS 2007 04). Eur J Cancer. 2013;49(18):3856-3862.

49. Janssen JM, Zwaan CM, Schellens JHM, Beijnen JH, Huitema ADR. Clinical trial simulations in paediatric oncology: a feasibility study from the Innovative Therapies for Children with Cancer Consortium. Eur J Cancer. 2017;85:78-85.

50. Cohen KJ, et al. Temozolomide in the treatment of children with newly diagnosed diffuse intrinsic pontine gliomas: a report from the Children's Oncology Group. Neuro-oncology. 2011;13(4):410-416.

51. Gabrilovich DI. Myeloid-derived suppressor cells. Cancer Immunol Res. 2017;5(1):3-8.

52. Wickham H, Grolemund G. R for data science: import, tidy, transform, visualize, and model data. O'Reilly Media; 2017. 